\title{
Chord progressions selection based on song audio features
}

\author{
Noelia Rico ${ }^{1}$ and Irene Díaz ${ }^{1}$ \\ Computer Science Department at University of Oviedo, Spain \\ noripa@gmail.com, sirene@uniovi.es
}

\begin{abstract}
A chord progression is an essential building block in music. In the field of music theory is usually assumed that these progressions influence the mood, emotion, genre or other critical aspects of the songs, and also in the perception that they will cause on the listener. Therefore, it is natural to think that musical and audio features of a track should be related to its chord progressions. Choosing carefully these progressions when it comes the time of creating a new song, is a fundamental aspect depending on the feelings we want to evoke on the listener. Also, two songs can be considered alike or classified into the same emotions or genres if they use the same chord progressions. Many music classification studies are presented nowadays, but none of them take into account chord progressions, probably due to the lack of this kind of data. In this paper, classification algorithms are used to illustrate the influence of the songs' features when it comes to pick up chord progressions to create a new song.
\end{abstract}

Keywords: Music Information Retrieval, Chord Progressions, Data Mining, Machine Learning, Classification

\section{Introduction}

It is generally accepted that music makes us feel a wide range of emotions. The mechanisms through which music evokes feelings and impacts in our brain is a vast field of research with a lot of unanswered questions.

On many occasions, the songs can be stirring even though they do not have any lyrics, and this is why music is considered an international language. In these cases, the songs' emotions are purely related to their structure [1]. Structural features of a song include rhythm, musical nuances, melody and the chords over which these melodies are settled. The same song can convey entirely different emotions after changing its chord progressions. Thus, listeners' feelings after hearing the modified song could be completely opposite. Those changes might also modify other aspects of the song such as its energy or popularity.

In the field of music regarding chord progressions, previous work has only focused on the analysis of music scores (most of the time using a MIDI file) in order to automatize the process of generating new music. Some of the previous research in this discipline is based on the identification of the chords and the 
progressions themselves [2]. Others, center their efforts in using machine learning techniques to generate music chords progression, based on songs that are used to build the model and taking into account only their musical score [3-5]. Related work in this field of study combines musical features with the song lyrics [6] to classify the songs or use specific musical features to divide the songs into emotions [7].

However, there has been little discussion on how the audio features and the impact of the song on the listener are correlated with the chord progressions found in the song. One question that needs to be asked is how the chosen progressions for the composition of each song influence some musical aspects not directly related to the music score, such as the energy that the songs transmit or their future popularity.

This paper proposes a methodology to find the audio features of a track affecting their chord progressions. These audio features can be described as the musical features of the song in addition to some other attributes about its impact on the listener.

This paper is divided into five sections. Section 2 describes the dataset, explaining its creation and describing its features in detail. Section 3 gives an overview of the methods used to build the models. In section 4 the results of the machine learning process are analyzed. Finally, the last section discusses the evaluation and possible application of the classification process and future work is proposed.

\section{Datasets creation}

One of the most complex tasks in this work is getting the data set. For this purpose, data from Hook Theory [8] and Spotify [9] websites are considered. The information about a song provided by these two different sources is supposed to be related one to each other. Thus, the two datasets obtained are joined in order to perform a better prediction. Then, this section first details how to obtain information from Hook Theory and Spotify websites and how to put them together.

\subsection{Hook Theory}

Hook Theory [8] is a popular website in the music field that allows their users to analyze songs regarding chord progressions and share their analysis. It is designed to be used by people who are learning how to compose new music, integrating an interactive interface for people who do not know anything about music theory. For this purpose, this website includes a section showing the most popular chord progressions in music of all styles, ages and genres. Those "most popular progressions" are grouped into categories, to make easier the learning process for the amateur composers.

Using the API [10] of the website we have gathered all the songs that contain a specific song progression. The chord progressions categories that have been 
used for this work are shown in Table 1. The second column describes the name used on the website to identify each progression and the first one indicates the group where the progression is wrapped, regarding how challenging is to use it when composing a song. The third column represents the chord progression itself and the fourth column shows the name that has been given to each progression in this project, and therefore the one which is used to identify the variable representing the progression in the final dataset.

The third column represents the chord progression as the succession of musical chords expressed by Roman numerals, as is common in Classical music theory. Following the standard, the capital letters are used to express whether the chord is major or minor. The progressions with lower difficulty are built with chords corresponding to basic degrees in the scale $(I, V, i i i \ldots)$. Whilst difficulty increases, the chords evolve to more complex constructions, and therefore the appearance of indexes becomes popular to indicate the inversion of the chord.

\begin{tabular}{llccc}
\hline Difficulty & Name & Chord Progression & Dataset variable \\
\hline \multirow{5}{*}{ Beginner } & Most popular progression & $I-V-v i-I V$ & most.popular \\
& Pachelbel's progression & $I-V-v i-i i i$ & pachelbel \\
& Effective in all genres & $v i-V-I V-V$ & effective.all.genres \\
& Those magic changes & $I-v i-I V-V$ & magic.changes \\
& Gaining popularity & $I-I V-v i-V$ & gaining.popularity \\
& Timeless & $I-V-I V-V$ & timeless \\
\hline \multirow{5}{*}{ Intermediate I } & The cadential ${ }_{4}^{6}$ & $I_{4}^{6}-V$ & cadential64 \\
& Stepwise bass down & $I-V^{6}-v i$ & stepwise.bass.down \\
& Stepwise bass up & $I-i i^{7}-I^{6}$ & stepwise.bass.up \\
& The newcomer & $I-i i i_{4}^{6}-v i$ & newcomer \\
& $I^{6}$ as a precadence & $I V-I^{6}-V$ & I.as.precadence \\
& Simple yet powerful & $I V-I^{6}-i i$ & simple.yet.powerful \\
\hline \multirow{5}{*}{ Intermediate II } & Chord pleased the Lord & $V-V^{6} / v i-v i$ & chord.pleased.Lord \\
& Expanding with V/vi & $I-V / v i$ & expanding.V.vi \\
& $v i_{2}^{4}$ as a passing chord & $v i-v i_{2}^{4}-I V$ & vi42.passing.chord \\
& Secondary dominant & $V{ }^{7} / I V-I V$ & secondary.dominant \\
& Applied vii & $v i i^{\circ} / v i-v i$ & applied.vii \\
\hline Cadencing in style & $I V-i v-I$ & candencing.in.style \\
Advanced & A mixolydian cadence & $b V I I-I V-I$ & mixolydian.cadence \\
& Using b VI to set up a V & $b V I-V$ & bVI.to.V \\
& Cadencing via b VII & $b V I I-I$ & cadencing.bVII \\
\hline
\end{tabular}

Table 1: Attributes obtained from the Hook Theory API

Once all the songs have been collected, the dataset is build using a row to represent each song. Songs are identified by two columns, which are title and artist, and the dataset contains 21 more columns, each one associated to 
a different chord progression. If the song of the row $i$ contains the progression $j$, the cell $_{i j}$ has the value 1 , otherwise its value will be 0 . The final dataset extracted from Hook Theory website [8] contains 1990 songs characterized by 23 attributes.

\subsection{Spotify}

The Spotify API fetches data [9] of all the available songs in the Spotify music catalog. To construct the second dataset based on Spotify, the songs contained in HookTheory dataset are considered. For each song in the hooktheory dataset, a search with the Spotify API is made using the primary key of the songs (artist and title). If the track is found, their features are extracted and the song is added to this new dataset. Among all the data available in the Spotify API, only the attributes that have been considered useful for this research will be included. Some of these features are strictly pulled out of the song's musical attributes like tempo or key, but others are calculated from its audio features like valence, or based on their reproductions on Spotify like popularity. These features are used as predictors of the final dataset. A thorough description of the attributes can be found in Table 2 .

\subsection{Features and class of the final dataset}

Using the data obtained from both Spotify and Hook Theory websites, different features characterizing 1990 songs have been collected. Features extracted from Spotify (see 2) represent the attributes associated with each song, while features obtained from Hook Theory website represent the class to learn. Thus, Features extracted from Spotify will be used to predict each chord progression as a binary problem (whether the song contains a chord progression or not).

Note that 21 different datasets will be created, each one linked with a different chord prediction 1 . To sum up, 21 binary classification problems will be solved, taking into account the Spotify features as predictor variables. All the problems are independent one to each other, but they have the same structure. For each problem, a different binary class variable will be considered. The aim of each problem is to classify the songs into two possible categories, 0 or 1 . The value will be 1 if the song uses the chord considered as the class variable for that concrete problem and 0 otherwise.

\section{Learning Procedure}

\subsection{Methods}

The classification will be made using $\mathrm{R}$, and more precisely the caret [11] package. Six different algorithms will be used to build the models. This will allow us to make a comparison about the performance and the final results:

- rpart: PART tree 


\begin{tabular}{|c|c|c|}
\hline feature & type & description \\
\hline artist & $\operatorname{chr}$ & used as id of the song together with title \\
\hline title & $\operatorname{chr}$ & used as id of the song together with artist \\
\hline popularity & int & $\begin{array}{l}\text { The value will be between } 0 \text { and } 100 \text { expressing the pop- } \\
\text { ularity of the track, with } 100 \text { being the most popular. } \\
\text { Songs that are being played a lot now will have a higher } \\
\text { popularity than songs that were played a lot in the past }\end{array}$ \\
\hline explicit & Factor & $\begin{array}{l}\text { Two possible levels: TRUE/FALSE. Whether or not the } \\
\text { track has explicit lyrics }\end{array}$ \\
\hline danceability & num & $\begin{array}{l}\text { Value between } 0.0 \text { and } 1.0 \text { describing how suitable a track } \\
\text { is for dancing based on a combination of musical elements } \\
\text { (tempo, rhythm stability, beat strength, overall regular- } \\
\text { ity). A value of } 0.0 \text { is least danceable and } 1.0 \text { is most } \\
\text { danceable }\end{array}$ \\
\hline energy & num & $\begin{array}{l}\text { Value between } 0.0 \text { to } 1.0 \text { that represents a perceptual } \\
\text { measure of intensity and activity. Perceptual features } \\
\text { contributing to this attribute include dynamic range, per- } \\
\text { ceived loudness, timbre, onset rate, and general entropy }\end{array}$ \\
\hline key & Factor & $\begin{array}{l}12 \text { possible levels. The key track is in. Integers map to } \\
\text { pitches using standard Pitch Class notation }\end{array}$ \\
\hline loudness & num & $\begin{array}{l}\text { The overall loudness of a track in decibels }(\mathrm{dB}) \text {. Loudness } \\
\text { values are averaged across the entire track. Values typical } \\
\text { range between }-60 \text { and } 0 \mathrm{db}\end{array}$ \\
\hline speechiness & num & $\begin{array}{l}\text { Value between } 0.0 \text { to } 1.0 \text {. Detects the presence of spoken } \\
\text { words in a track. The more exclusively speech-like the } \\
\text { recording the closer to } 1.0 \text { the attribute value }\end{array}$ \\
\hline acousticness & num & $\begin{array}{l}\text { A confidence measure from } 0.0 \text { to } 1.0 \text { of whether the track } \\
\text { is acoustic. } 1.0 \text { represents high confidence the track is } \\
\text { acoustic }\end{array}$ \\
\hline instrumentalness & num & $\begin{array}{l}\text { Value between } 0.0 \text { and } 1.0 \text {. The closer the instrumental } \\
\text { ness value is to } 1.0 \text {, the greater likelihood that the track } \\
\text { contains no vocal content. Vocals like "Ooh" and "aah" } \\
\text { are treated as instrumental in this context }\end{array}$ \\
\hline valence & num & $\begin{array}{l}\text { A measure from } 0.0 \text { to } 1.0 \text { describing the musical posi- } \\
\text { tiveness conveyed by a track. Tracks with high valence } \\
\text { sound more positive while tracks with low valence sound } \\
\text { more negative }\end{array}$ \\
\hline tempo & num & $\begin{array}{l}\text { The overall estimated tempo of a track in beats per } \\
\text { minute (BPM). In musical terminology, tempo is the } \\
\text { speed or pace of a given piece and derives directly from } \\
\text { the average beat duration. }\end{array}$ \\
\hline duration_ms & int & The duration of the track in milliseconds \\
\hline time_signature & int & $\begin{array}{l}\text { An estimated overall time signature of a track. The time } \\
\text { signature (meter) is a notational convention to specify } \\
\text { how many beats are in each bar (or measure) }\end{array}$ \\
\hline mode & Factor & $\begin{array}{l}\text { Mode indicate the modality (major or minor) of a track, } \\
\text { the type of scale from which its melodic content is de- } \\
\text { rived. Major is represented by } 1 \text { and minor is } 0\end{array}$ \\
\hline
\end{tabular}

Table 2: Features fetched from the Spotify API 
- glm: Generalized Linear Model

- nb: Naive Bayes

- svmLinear: Support Vector Machines with Linear Kernel

- svmRadial: Support Vector Machines with Radial Basis Function Kernel

- ranger: Random Forest

The performance and utility of the above-detailed Machine Learning methods in different domains ([12], [13], [14], [15], [16]) allows us to use them for predicting chord progressions. Different methods have been selected to cover different learning paradigms.

Method glm [?] fits generalized linear models, specified by giving a symbolic description of the linear predictor and a description of the error distribution. It is suitable for binary classification problems.

Tree modeling is performed through rpart [17] and ranger [18] (upgrade of the classical randomForest). rpart carries out a CART (Classification and regression trees) modeling. First of all, it grows the (classification in this case) tree until one of the possible stop conditions are reached: the number of observations in a node is under a threshold, the minimum cost complexity factor cannot be reached when attempting to do a split or the tree has reached the maximum depth allowed. Once the tree has been grown, the method examines the results and if over fitting is detected, the tree is pruned to find an optimal point between over fitting and under fitting. These methods split the data based on how the creation of sub-nodes increases the homogeneity of those resultant sub-nodes. Random forests technique is based on trees too but it improves predictive accuracy by generating not one but a large number of bootstrapped trees using random samples of variables for each iteration, classifying a case using each tree in this newest created forest, and deciding the final predicted outcome by combining the results across all of the trees.

Naive Bayes is based on the Bayes Theorem and although all the predictors are assumed to be independent within each class label, it gives excellent results for many real problems. The predictor variables are handled by assuming that they follow a Gaussian distribution, given the class label. The outcome variable is predicted using the probabilities of each attribute when $\mathrm{nb}$ [19] is applied. Support Vector Machines [20] performs classification looking for the hyperplane that maximizes the margin between the classes closest points. When a linear separator cannot be found, data points are projected into a higher dimensional space where the data become linearly separable. This projection is made by kernel techniques. Two different methods with linear svmLinear and non-linear svmRadial kernel have been chosen

The above introduced methods have been applied considering the following settings:

- Method configuration: Default parameter settings provided by caret package in order to perform a first approach of how the datasets behave with each algorithm.

- Validation: Stratified split into training (80\%) and test (20\%). The training set will be used to build the model and the test set to evaluate it. 
- Training: 10 fold cross validation with 3 repetitions has been used for training the models. In this 10 fold cross validation, all the samples in the dataset were grouped in 10 different sets, using one of them for testing while keeping the remaining for training. This process is repeated three times.

Note that most of the datasets are imbalanced $(30 \%(1) / 70 \%(0))$. Thus, different sampling strategies are tested and the resulting datasets represent the input to each one of the learning methods. For each of the six algorithms, four different models have been built according to a different sampling strategy (over, down, ROSE and SMOTE [11]). For each algorithm, the model yielding the best results on the test set will be selected as the best model. The evaluation of this previously unseen instances of the test set let us compare the real performance of the final models. Algorithm 1 shows the whole procedure.

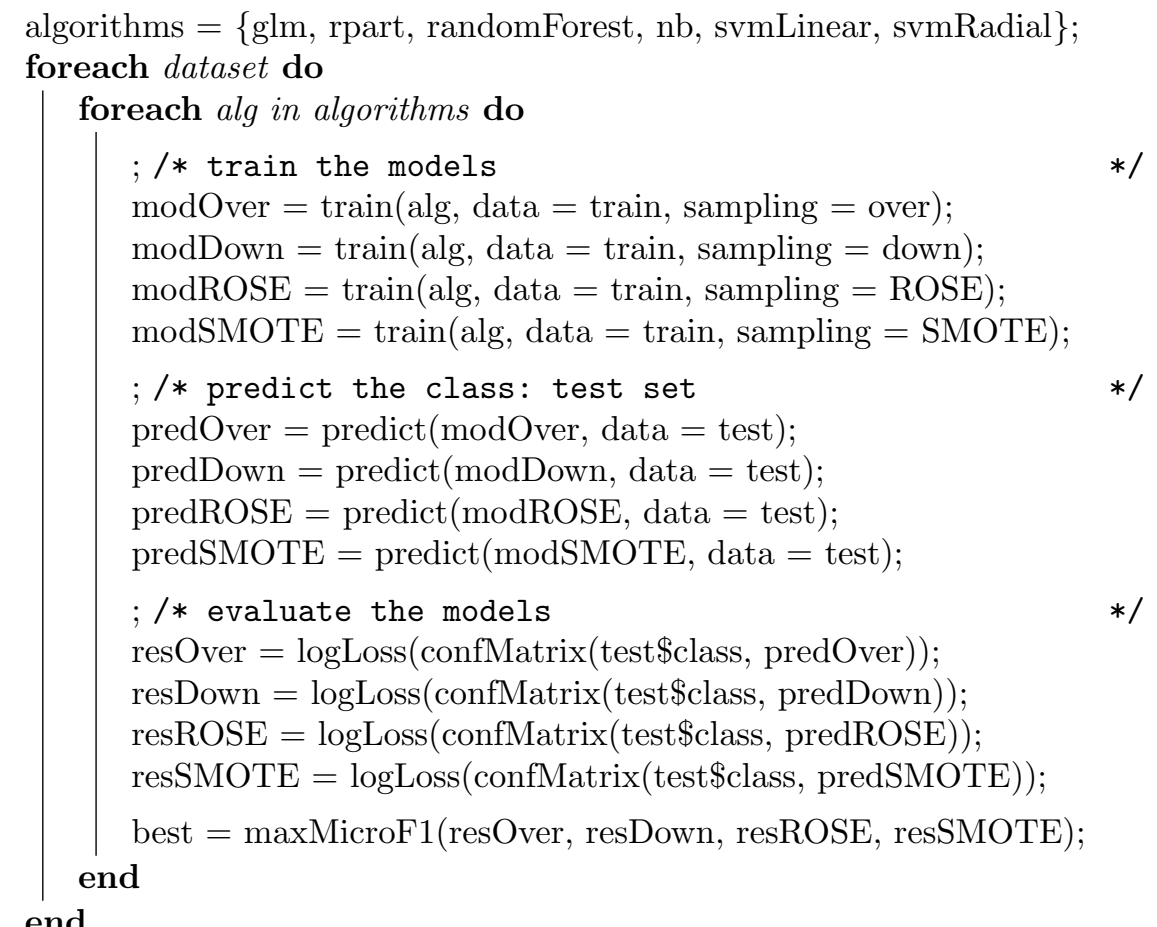

Algorithm 1: Build and select the best model for each dataset

\section{$3.2 \quad$ Metrics}

As explained before, some of the datasets' class are imbalanced. In addition to Accuracy (A), the micro averaged version of the well known metrics Precision $(\mathrm{P})$, Recall $(\mathrm{R})$ and $F_{1}$ [11] is selected together with logLoss [21]. Considering that TP is the number of examples correctly classified as the positive class, TN is the number of examples correctly classified as the negative class, FP is the 
number of examples wrongly classified as the positive class and FN the number of examples wrongly classified as the negative class, A, P, R and $F_{1}$ are defined as follows.

$$
\begin{aligned}
A=\frac{T P+T N}{T P+T N+F P+F N} & P=\frac{T P}{T P+F P} \\
R=\frac{T P}{T P+F N} & F_{1}=2 \times \frac{P \times R}{P+R}
\end{aligned}
$$

LogLoss quantifies the accuracy of a classifier by penalizing wrong classifications. Minimizing the Log Loss is equivalent to maximizing the accuracy of the classifier, but there is a subtle difference. In order to calculate the Log Loss, the classifier must assign a probability $\left(p_{i}\right)$ to each class rather than simply yielding the most likely class. It is defined below.

$$
\text { LogLoss }=-\frac{1}{N} \sum_{i=1}^{N}\left[y_{i} \log p_{i}+\left(1-y_{i}\right) \log \left(1-p_{i}\right)\right]
$$

\section{Results}

\begin{tabular}{ccccccc}
\hline progression & rpart & glm & nb & svmL & svmR & rf \\
\hline effective.all.genres & 0.74825 & 0.69578 & 0.73521 & 0.69849 & 0.74934 & $\mathbf{0 . 7 9 1 9 3}$ \\
gaining.popularity & 0.84074 & 0.72467 & $\mathbf{0 . 8 3 9 5 6}$ & 0.75179 & 0.82217 & 0.80793 \\
magic.changes & $\mathbf{0 . 8 6 8 3 9}$ & 0.76379 & 0.29982 & 0.72214 & 0.82843 & 0.86310 \\
most.popular & 0.67680 & 0.65479 & 0.65490 & 0.67097 & $\mathbf{0 . 6 9 0 7 5}$ & 0.68522 \\
pachelbel & 0.74137 & 0.86923 & 0.83064 & 0.87494 & $\mathbf{0 . 9 0 2 7 0}$ & 0.75444 \\
timeless & 0.73474 & 0.75117 & 0.41014 & 0.68525 & $\mathbf{0 . 8 2 3 1 8}$ & 0.56898 \\
cadential64 & 0.83682 & 0.81701 & 0.62892 & 0.82006 & 0.88455 & $\mathbf{0 . 8 9 9 0 8}$ \\
I.as.precadence & $\mathbf{0 . 9 5 2 3 4}$ & 0.79190 & 0.83517 & 0.75889 & 0.81397 & 0.76771 \\
newcomer & 0.77865 & 0.81385 & 0.68669 & 0.82314 & 0.74944 & $\mathbf{0 . 9 1 1 1 1}$ \\
simple.yet.powerful & $\mathbf{0 . 9 4 0 6 3}$ & 0.76104 & 0.51293 & 0.80395 & 0.91206 & 0.92766 \\
stepwise.bass.down & 0.71599 & 0.69285 & 0.76457 & 0.70821 & 0.72704 & $\mathbf{0 . 7 8 1 0 9}$ \\
stepwise.bass.up & 0.48695 & 0.84957 & 0.63792 & 0.89845 & $\mathbf{0 . 9 2 0 0 8}$ & 0.82191 \\
applied.vii & 0.82918 & 0.80775 & 0.81376 & 0.83822 & $\mathbf{0 . 8 8 3 8 9}$ & 0.74348 \\
chord.pleased.Lord & $\mathbf{0 . 8 8 3 9 9}$ & 0.85250 & 0.41050 & 0.82230 & 0.78972 & 0.63735 \\
expanding.V.vi & 0.81187 & 0.70590 & $\mathbf{0 . 8 4 8 2 1}$ & 0.72716 & 0.81105 & 0.80814 \\
secondary.dominant & 0.90437 & 0.88067 & 0.85573 & 0.88628 & $\mathbf{0 . 9 4 2 3 4}$ & 0.91330 \\
vi42.passing.chord & 0.89668 & 0.82953 & 0.86953 & 0.83557 & $\mathbf{0 . 9 1 2 3 3}$ & 0.82343 \\
bVI.to.V & 0.84705 & 0.85576 & 0.81376 & 0.83221 & $\mathbf{0 . 9 0 8 1 4}$ & 0.66306 \\
cadencing.bVII & 0.80866 & 0.73626 & $\mathbf{0 . 8 3 3 0 9}$ & 0.75970 & 0.82873 & 0.82798 \\
candencing.in.style & 0.84614 & 0.78441 & $\mathbf{0 . 9 3 8 0 2}$ & 0.82860 & 0.88073 & 0.93221 \\
mixolydian.cadence & 0.90957 & 0.79328 & 0.49135 & 0.76125 & 0.84047 & $\mathbf{0 . 9 0 9 5 7}$ \\
\hline
\end{tabular}

Table 3: $F_{1}$ for each model on test set 


\begin{tabular}{rcccccc}
\hline progression & rpart & glm & nb & svmL & svmR & rF \\
\hline effective.all.genres & 2.29080 & 0.61709 & 1.06604 & 0.64638 & 0.54090 & $\mathbf{0 . 5 0 8 1 9}$ \\
gaining.popularity & 2.29586 & 0.59268 & 0.68104 & 0.55032 & 0.56741 & $\mathbf{0 . 5 3 0 5 2}$ \\
magic.changes & 0.45711 & 0.88835 & 5.69869 & 0.58640 & 0.45529 & $\mathbf{0 . 4 1 5 3 6}$ \\
most.popular & 0.63065 & 0.61723 & 0.92931 & 0.61045 & 0.61680 & $\mathbf{0 . 5 9 2 2 6}$ \\
pachelbel & 0.73712 & 0.57187 & 2.20434 & 0.49506 & $\mathbf{0 . 4 2 2 3 9}$ & 0.66887 \\
timeless & 1.95744 & 0.85849 & 4.27938 & 0.64156 & $\mathbf{0 . 4 5 3 8 9}$ & 0.74188 \\
cadential64 & 0.55121 & 0.66113 & 3.76041 & 0.51237 & $\mathbf{0 . 3 6 3 7 0}$ & 0.39110 \\
I.as.precadence & 0.76120 & 0.63053 & 1.47563 & $\mathbf{0 . 5 6 6 0 8}$ & 0.61571 & 0.62741 \\
newcomer & 0.67570 & 1.00073 & 2.20718 & 0.49264 & 0.80781 & $\mathbf{0 . 3 9 6 9 6}$ \\
simple.yet.powerful & 1.38791 & 0.73773 & 2.11598 & 0.60028 & $\mathbf{0 . 3 2 2 3 8}$ & 0.39084 \\
stepwise.bass.down & 2.03425 & 0.64715 & 0.96973 & 0.64085 & 0.61982 & $\mathbf{0 . 6 0 7 2 5}$ \\
stepwise.bass.up & 1.14947 & 0.50541 & 1.76185 & 0.51010 & $\mathbf{0 . 3 4 4 4 7}$ & 0.60753 \\
applied.vii & 0.69399 & 0.61890 & 1.22321 & 0.58839 & $\mathbf{0 . 4 0 0 9 1}$ & 0.66001 \\
chord.pleased.Lord & $\mathbf{0 . 3 9 4 9 3}$ & 2.57653 & 10.74308 & 0.46906 & 0.85578 & 1.33383 \\
expanding.V.vi & 0.55673 & 0.66775 & $\mathbf{0 . 4 7 1 8 3}$ & 0.65301 & 0.49104 & 0.59195 \\
secondary.dominant & 0.36368 & 0.39952 & 0.77317 & 0.35223 & $\mathbf{0 . 1 7 5 5 4}$ & 0.26221 \\
vi42.passing.chord & 0.66081 & 0.62432 & 0.88796 & 0.50365 & $\mathbf{0 . 3 4 0 0 7}$ & 0.58048 \\
bVI.to.V & 1.78251 & 0.51041 & 3.98525 & 0.47484 & $\mathbf{0 . 3 2 7 2 2}$ & 0.72091 \\
cadencing.bVII & 0.78476 & 0.60006 & 0.72928 & 0.56762 & $\mathbf{0 . 4 9 9 1 1}$ & 0.45051 \\
candencing.in.style & 0.58210 & 0.61831 & 0.53075 & 0.44616 & 0.38625 & $\mathbf{0 . 3 8 4 1 1}$ \\
mixolydian.cadence & 1.59762 & 0.63714 & 3.21393 & 0.53388 & 0.52423 & $\mathbf{0 . 4 0 1 0 9}$ \\
\hline & & & & & &
\end{tabular}

Table 4: LogLoss of each model on the test set

In this section, the aforementioned experiments are analyzed. Table 3 shows the $F_{1}$ obtained for each binary problem and each different machine learning method. Table 4 contains the LogLoss value for the experiments whose $F_{1}$ is shown in Table 3.

Note that $F_{1}$ is often high. However, the highest $F_{1}$ is obtained with the combination of SMOTE resampling method and svmRadial. In particular, the results with highest $F_{1}$ and lowest logLoss are obtained when model is trained with svmRadial, being this the best for the $38 \%$ of the progressions, followed by the method ranger, which achieves the best results for the $33 \%$ of the progressions. We recommend the use of svmRadial because it has lower computational cost, and therefore it is quicker building the model. Table 5 shows the most important variables used for building the models. It should be noticed how the valence appears in the top four important variables for most of the models. Other features such as danceability, valence, loudness or popularity together with other strictly musical related like instrumentalness or key, stand out from the list of important variables used to build the models.

The evidence from this study points towards the idea that information gathered from Spotify could be useful for helping us decide which chord progressions should be used when a new song is being created. 


\begin{tabular}{rcccc}
\hline \multicolumn{1}{c}{ progression } & first & second & third & fourth \\
\hline effective.all.genres duration_ms & acousticness & energy & instrumentalness \\
gaining.popularity duration_ms & loudness & valence & speechiness \\
magic.changes & tempo & acousticness & danceability & valence \\
most.popular & loudness & duration_ms & valence & popularity \\
pachelbel & key8 & valence & mode1 & instrumentalness \\
timeless & mode1 & valence & acousticness & key1 \\
cadential64 acousticness & speechiness & valence & duration_ms \\
I.as.precadence & valence & acousticness & danceability & duration_ms \\
newcomer acousticness & mode1 & valence & danceability \\
simple.yet.powerful & valence & danceability & popularity & instrumentalness \\
stepwise.bass.down & valence & popularity & acousticness & duration_ms \\
stepwise.bass.up acousticness & energy & loudness & key10 \\
applied.vii acousticness instrumentalness & energy & valence \\
chord.pleased.Lord & loudness & speechiness & popularity & energy \\
expanding.V.vi & speechiness & valence & loudness & instrumentalness \\
secondary.dominant & energy & loudness & acousticness & valence \\
vi42.passing.chord & key6 & acousticness & loudness & valence \\
bVI.to.V & loudness & valence & energy & danceability \\
cadencing.bVII & popularity & loudness & instrumentalness & acousticness \\
candencing.in.style acousticness & loudness & popularity & instrumentalness \\
mixolydian.cadence & loudness & instrumentalness & acousticness & valence \\
\hline
\end{tabular}

Table 5: Most important variables of each model

\section{Conclusions and Future Work}

This paper presents a method to improve knowledge about how useful chord progressions can be in other music classification problems, which aims to classify songs into emotions or genres.

The method presented in this work has many interesting applications in the composition of new music. If done manually, the work of analyzing all the chord progressions in a music score is not an easy task. It requires time and a great knowledge of music theory. Automating this process is not trivial either, it has a high computing cost, and the results are not always satisfactory.

These results represent an excellent initial step toward a new field of application. The principal advantages are the creation of new songs based on the features of similar and already existing songs, without the need to analyze their structure and taking into account not only their musical features but aspects of the tracks as their energy or valence.

We are currently working on the multi-label version of this problem, that can address the issue of creating a new track based on a bunch of songs we want it to be similar to. Given as input existing songs, the classification model will return the list of chord progressions that should and should not be used to create the new composition.

Further work will look into the lyrics of the songs, in order to perform a sentiment analysis process to explore the correlation between the Spotify musical 
features, the chord progressions and the emotions the song intends to cause on the listener, based on the vocabulary they are using.

\section{Acknowledgments}

This research has been funded by the Spanish MINECO project TIN2017-87600P.

\section{References}

1. Juslin, P.N., Sloboda, J.: Handbook of Music and Emotion: Theory, Research, Applications. Oxford University Press (2011)

2. Cho, Y.H., Lim, H., Kim, D.W., Lee, I.K.: Music emotion recognition using chord progressions. In: Systems, Man, and Cybernetics (SMC), 2016 IEEE International Conference on, IEEE (2016) 002588-002593

3. Stamatatos, E., Widmer, G.: Automatic identification of music performers with learning ensembles. Artificial Intelligence 165(1) (2005) 37 - 56

4. Arutyunov, V., Averkin, A.: Genetic algorithms for music variation on genom platform. Procedia Computer Science 120 (2017) 317 - 324 9th International Conference on Theory and Application of Soft Computing, Computing with Words and Perception, ICSCCW 2017, 22-23 August 2017, Budapest, Hungary.

5. Costa, Y.M., Oliveira, L.S., Silla, C.N.: An evaluation of convolutional neural networks for music classification using spectrograms. Applied Soft Computing 52 (2017) $28-38$

6. Hu, X., Downie, J.S.: Improving mood classification in music digital libraries by combining lyrics and audio. In: Proceedings of the 10th Annual Joint Conference on Digital Libraries. JCDL '10, New York, NY, USA, ACM (2010) 159-168

7. Martin-Gómez, L., Navarro-Cáceres, M.: Applying data mining for sentiment analysis in music. In De la Prieta, F., Vale, Z., Antunes, L., Pinto, T., Campbell, A.T., Julián, V., Neves, A.J., Moreno, M.N., eds.: Trends in Cyber-Physical Multi-Agent Systems. The PAAMS Collection - 15th International Conference, PAAMS 2017, Cham, Springer International Publishing (2018) 198-205

8. Famous-Chord-Progressions: https://www.hooktheory.com/theorytab/commonchord-progressions (January 2018)

9. Spotify: https://developer.spotify.com/web-api/get-audio-features/ (January 2018)

10. HookTheory-API: https://www.hooktheory.com/api/trends/docs (January 2018)

11. Kuhn, M.: Building predictive models in $\mathrm{R}$ using the caret package. Journal of Statistical Software 28(5) (11 2008) 1-26

12. Villar, J.R., Chira, C., Sedano, J., González, S., Trejo, J.M.: A hybrid intelligent recognition system for the early detection of strokes. Integrated Computer-Aided Engineering 22(3) (2015) 215-227

13. Herrero, Á., Sedano, J., Baruque, B., Quintián, H., Corchado, E., eds.: 10th International Conference on Soft Computing Models in Industrial and Environmental Applications, SOCO 2015, Burgos, Spain, June 2015. Volume 368 of Advances in Intelligent Systems and Computing., Springer (2015)

14. Troiano, L., Rodríguez-Muñiz, L.J., Ranilla, J., Díaz, I.: Interpretability of fuzzy association rules as means of discovering threats to privacy. Int. J. Comput. Math. 89(3) (2012) 325-333 
15. Gil-Pita, R., Ayllón, D., Ranilla, J., Llerena-Aguilar, C., Díaz, I.: A computationally efficient sound environment classifier for hearing aids. IEEE Trans. Biomed. Engineering 62(10) (2015) 2358-2368

16. Montañés, E., Quevedo, J.R., Díaz, I., Ranilla, J.: Collaborative tag recommendation system based on logistic regression. In: Proceedings of ECML PKDD (The European Conference on Machine Learning and Principles and Practice of Knowledge Discovery in Databases) Discovery Challenge 2009, Bled, Slovenia, September 7, 2009. (2009)

17. Chambers, J.M.: Statistical Models in S. CRC Press, Inc., Boca Raton, FL, USA (1991)

18. Breiman, L., Friedman, J., Olshen, R., Stone, C.: Classification and Regression Trees. Wadsworth and Brooks, Monterey, CA (1984)

19. Wright, M.N., Ziegler, A.: ranger: A fast implementation of random forests for high dimensional data in C++ and R. Journal of Statistical Software 77(1) (2017) $1-17$

20. Rish, I.: An empirical study of the naive bayes classifier. In: IJCAI 2001 workshop on empirical methods in artificial intelligence. Volume 3., IBM New York (2001) $41-46$

21. Cortes, C., Vapnik, V.: Support-vector networks. Machine Learning 20(3) (Sep 1995) 273-297

22. Masnadi-shirazi, H., Vasconcelos, N.: On the design of loss functions for classification: theory, robustness to outliers, and savageboost. In Koller, D., Schuurmans, D., Bengio, Y., Bottou, L., eds.: Advances in Neural Information Processing Systems 21. Curran Associates, Inc. (2009) 1049-1056 